\title{
BMJ Open Study protocol: a prospective cohort on non-communicable diseases among primary healthcare users living in Kosovo (KOSCO)
}

\author{
Katrina Ann Obas (D) ,1,2 Jana Gerold, ${ }^{2,3}$ Ariana Bytyçi-Katanolli, ${ }^{1,2}$ Naim Jerliu, ${ }^{4,5}$ \\ Marek Kwiatkowski, ${ }^{1,2}$ Qamile Ramadani, ${ }^{6}$ Shukrije Statovci, ${ }^{7}$ Manfred Zahorka, ${ }^{2,3}$ \\ Nicole Probst-Hensch ${ }^{1,2}$
}

To cite: Obas KA, Gerold J, Bytyçi-Katanolli A, et al. Study protocol: a prospective cohort on non-communicable diseases among primary healthcare users living in Kosovo (KOSCO). BMJ Open 2020;10:e038889. doi:10.1136/ bmjopen-2020-038889

- Prepublication history for this paper is available online. To view these files, please visit the journal online (http://dx.doi org/10.1136/bmjopen-2020038889).

Received 31 March 2020 Revised 08 August 2020 Accepted 20 August 2020
Check for updates

(C) Author(s) (or their employer(s)) 2020. Re-use permitted under CC BY-NC. No commercial re-use. See rights and permissions. Published by BMJ.

For numbered affiliations see end of article.

Correspondence to Dr Nicole Probst-Hensch; nicole.probst@swisstph.ch

\section{ABSTRACT}

Introduction With the lowest life expectancy in the Balkans, underlying causes of morbidity in Kosovo remain unclear due to limited epidemiological evidence. The goal of this cohort is to contribute epidemiological evidence for the prevention and control of non-communicable diseases such as depression, hypertension, diabetes and chronic respiratory disease in Kosovo as the basis for policy and decision-making, with a spotlight on the relationships between non-experimental primary healthcare (PHC) interventions and lifestyle changes as well as between depression and the course of blood pressure.

Methods and analysis PHC users aged 40 years and above were recruited consecutively between March and October 2019 from 12 main family medicine centres across Kosovo. The data collected through interviews and health examinations included: sociodemographic characteristics, social and environmental factors, comorbidities, health system, lifestyle, psychological factors and clinical attributes (blood pressure, height, weight, waist/hip/neck circumferences, peak expiratory flow and $\mathrm{HbA} 1 \mathrm{c}$ measurements). Cohort data were collected annually in two phases, approximately 6 months apart, with an expected total follow-up time of 5 years.

Ethics and dissemination Ethical approvals were obtained from the Ethics Committee Northwest and Central Switzerland (Ref. 2018-00994) and the Kosovo Doctors Chamber (Ref. 11/2019). Cohort results will provide novel epidemiological evidence on non-communicable diseases in Kosovo, which will be published in scientific journals. The study will also examine the health needs of the people of Kosovo and provide evidence for health sector decision-makers to improve service responsiveness, which will be shared with stakeholders through reports and presentations.

\section{INTRODUCTION}

Burden of non-communicable diseases in Kosovo The burden of disease in the Balkan region falls heaviest on Kosovo, suggested by a life expectancy of 72 years, ${ }^{1}$ which is lower than neighbouring countries such as Albania (78 years), Montenegro (77 years), Macedonia
Strengths and limitations of this study

- As the first prospective cohort covering different areas of Kosovo, the study will provide important evidence on the course of non-communicable diseases in a country with limited epidemiological evidence.

- The longitudinal study design will allow us to observe changes in non-communicable diseases and their determinants over time in individuals and analyse the temporal sequence of changes, thus providing stronger evidence in investigating causal relationships.

- Study results can be immediately applied in designing or adapting existing targeted behaviour change interventions by healthcare stakeholders.

- This study is not population based due to the recruitment scheme in primary healthcare facilities, which limits its generalisability and may overestimate the prevalence of health conditions; however, healthy persons are included in the study because primary healthcare patients visit centres for an array of conditions including general check-ups.

(76 years) and Serbia (76 years). ${ }^{1}$ It is a challenge, however, to get a better understanding of the main culprits of Kosovo's disease burden due in part to limited epidemiological evidence given the country's health information system is still in its initial developmental phase. $^{2}$

Although it is well known that noncommunicable diseases (NCDs) are the greatest contributor to health loss in the world, accounting for $61 \%$ (or 1.5 billion) of disability-adjusted life years (DALY), only a few estimates on NCDs in Kosovo are available. A national population-based study conducted in 2010 in Kosovo of adults over the age of $65(n=1890)$ indicated that the most common self-reported NCDs were cardiovascular diseases (CVDs), with a prevalence of 
$63 \%$, followed by stomach and liver disease $(21 \%)$, then diabetes mellitus (DM; $18 \%){ }^{3}$

CVDs are a major health concern globally, accounting for 353 million DALYs (14.8\% of all DALYs globally), over 471 million prevalent cases and 17.6 million deaths annually. ${ }^{4}$ The situation is dire in the Balkans, where the burden of CVDs is nearly double that of the global prevalence (27.7\% of all DALYs for the Balkans). CVDs include coronary artery disease, cardiomyopathy, cerebrovascular disease, peripheral vascular disease, rheumatic heart disease, arrhythmias and endo/myocarditis. Acute CVDs events include myocardial infarctions and strokes. The Kosovo Agency of Statistics reports that CVDs were responsible for $57.9 \%$ of deaths in $2012 ; 18 \%$ of these occurring under the age of $60 .^{5}$

Although CVDs are the principal causes of death worldwide, mental disorders are now among the leading causes of disability. ${ }^{4}$ Among mental disorders, depression is the most common with over 300 million prevalent cases worldwide (4.4\% global prevalence). ${ }^{6}$ Depression results from a complex interaction of social, psychological and biological factors and is characterised by persistent sadness, loss of interest in activities a person normally enjoys and inability to carry out daily activities. People who have gone through adverse life events (unemployment, bereavement, psychological trauma) are more likely to develop depression as well as post-traumatic stress disorder (PTSD). PTSD differs from depression in that the person must have experienced a traumatic event and experiences intense, disturbing thoughts and feelings related to their trauma that last long after the event has ended.

Depression and PTSD in Kosovo have been studied more extensively in the past two decades as a result of the scientific interest to study psychological effects following the war in the late 1990s. One nationally representative study ( $\mathrm{n}=1161$ ) of persons aged 15 years or older found that $41.7 \%$ had moderate-to-severe depressive symptoms and $41.6 \%$ had severe anxiety, measured by the Hopkins Symptoms Checklist (HSCL) ${ }^{7}$ PTSD was present in $22 \%$ of respondents, measured by the Harvard Trauma Questionnaire, and was predictive of suicidal ideation which was measured with a suicidal ideation index created using items from the General Health Questionnaire and HSCL. ${ }^{7}$ Other studies, which focused on specific regions of the country or specific subgroups, found a prevalence of depression which ranged from $29.7 \%$ to $66.5 \% .^{8-11}$ It is clear that depression is common in Kosovo, far exceeding the global average. Some interpret the high rates of depression as an aftermath to the stressful conditions following the war. ${ }^{12}$

In summary, CVD and depression are among the NCDs which cause the greatest burden to global health and may be important starting points for NCDs research in Kosovo.

\section{NCD prevention and control in Kosovo}

Primary healthcare (PHC) plays a key role in the prevention and control of CVDs and other NCDs. ${ }^{13}$ Primary prevention includes interventions which avert the occurrence of disease, whereas secondary prevention includes interventions which stop or slow the progression of disease once it has started. ${ }^{14}$ Many PHC interventions aim to reduce common risk factors of NCDs, such as smoking, physical inactivity and poor diet, in both healthy people and patients with NCDs. In Kosovo, the PHC system is divided into three tiers: each municipality has one main family medicine centre (MFMC), several family medicine centres (FMC) and several family medicine ambulantas (FMA). MFMCs, which formed the basis for the recruitment of the study participants, are the largest facilities at the highest level of PHC, which offer more services, staff and medical equipment and therefore have a higher patient flow compared with the second level FMCs and third level FMAs.

The Accessible Quality Healthcare (AQH) implementation project, which is funded by the Swiss Agency for Development and Cooperation and led by the Swiss Tropical and Public Health Institute (Swiss TPH), started in 2016 and is now one of the prominent projects in Kosovo working within the PHC system. The AQH project has been devoted to working with local stakeholders to improve the quality of PHC in the public health sector through a health system strengthening approach, with a focus on the prevention of NCDs. The three project outcomes are as follows: (1) PHC providers deliver quality services that respond better to communities' needs, (2) health managers improve their performance in guiding service delivery towards continuous quality improvement, and (3) the population improves its health literacy and is empowered to demand the right to quality services and better access to care.

Health systems strengthening interventions implemented by the AQH project in Kosovo are broad and complex. One of the AQH interventions for improvement of PHC services is the implementation of service packages (SPs). This intervention aims to improve the quality of care by setting standards that should be provided at PHC facilities, based on the WHO 'Packages of Essential NonCommunicable Disease (PEN) Protocols, ${ }^{15}$ which have been adapted to the Kosovo context by national experts. The SPs ensure a continuum of care with the family physician in a gatekeeper role, where patients who are at risk of developing diabetes or hypertension, or those who have already been diagnosed are referred to a health educator for one-to-one motivational counselling sessions to facilitate behaviour change. Behaviour change is facilitated through lifestyle medicine, which is evidence-based practice of assisting individuals and families to adopt and sustain behaviours that can improve health and quality of life (QoL). Healthy behaviours could greatly influence future health and well-being, especially among patients with NCDs' ${ }^{16}$ In the long run, improving the health of populations means that individuals, communities and organisations need to change their behaviour to become healthier. ${ }^{17}$ The principal modifiable risk factors for CVDs and other NCDs include: tobacco use, an unhealthy diet 
and physical inactivity (which together result in obesity), hypertension, dyslipidaemia and diabetes. ${ }^{18}$ Prevention, management or reversal of the modifiable risk factors can be achieved through leading a healthier lifestyle. ${ }^{19}$

When considering the reduction of CVDs, the most damaging risk factor in terms of attributable DALYs is hypertension. ${ }^{4}$ Hypertension is defined as blood pressure of above 140/90 mm Hg according to the European Society of Cardiology. ${ }^{20}$ The prevalence of hypertension is available in a few Kosovar studies, but no data on hypertension control is yet available. One cross-sectional study $(\mathrm{n}=423$, mean age 51 years $)$ of two rural predominantly ethnic-Serb communities in Kosovo found a hypertension prevalence of $42 \%{ }^{21}$ Another cross-sectional study of PHC users ( $n=1793$, mean age 51 years) in the capital city of Pristina found a prevalence of hypertension at $33.6 \%$ (39\% in men and $29 \%$ in women). ${ }^{22}$ A third crosssectional study in 20 villages with a mixture of ethnic-Serbs and ethnic-Albanians found a hypertension prevalence of $30.6 \%$ (mean age men $=62$ years, women $=49$ years) ${ }^{23}$

The state of other risk factors for CVDs in Kosovo is even less well known. Although one study showed that $18 \%$ of older adults self-reported a diagnosis of $\mathrm{DM}^{3}{ }^{3}$ it is suspected that DM is highly underdiagnosed in Kosovo, indicating a large diagnostic gap. For example, another population study $(\mathrm{n}=423)$ conducted in 2006 assessing the prevalence of kidney disease (a positive family history for Balkan Endemic Nephropathy, mild proteinuria, alpha 1-microglobulinuria, eGFR $<60 \mathrm{~mL} / \mathrm{min} / 1.73$ $\mathrm{m}^{2}$, anaemia, low specific gravity of urine and reduced kidney length) in adults aged 18 years and older living in 2 Serbian settlements in the municipality of Rahovec found that $13 \%$ of participants had a previous diagnosis of diabetes but $21 \% \quad(n=89)$ still had a pathological glycaemia finding (fasting blood glucose $>6.1 \mathrm{mmol} / \mathrm{L}$ ) ${ }^{24}$ Although all residents aged 18 years and above in the two settlements were eligible to participate in the study, the methodology in recruitment was not specified. Some studies on physical activity are available on Kosovar adolescents ${ }^{25}$ but no evidence is available for adults. Similarly, evidence on tobacco use in Kosovo has been focused on school children and adolescents. However, a recent publication on the WHO Stepwise approach to surveillance (STEPS) survey of persons aged 15-64 years conducted in $2010(\mathrm{n}=6400)$ showed that $37 \%$ of men and $20 \%$ of women in Kosovo smoke. ${ }^{26}$ The prevalence increased with age until it dropped at age 45 . The results from the same STEPS survey on physical activity, diet and cholesterol have not yet been published. The AQH project conducted a population-based study in 12 municipalities, with the aim to collect primary data on project indicators for a baseline against which the impact of the project activities will be measured. The study found that $20.6 \%$ of respondents smoked, $15 \%$ had ever consumed alcohol and $46 \%$ did not meet WHO recommendations on physical activity. ${ }^{27}$

In summary, more information is needed about NCD risk factors in the context of Kosovo and the impact of current interventions aimed at their reduction for a better understanding of where to target PHC services.

\section{Mental disorders and their relationship with hypertension}

CVDs and mental disorders are among the most burdensome NCDs to global health. It is furthermore disconcerting that there is a well-established bidirectional relationship between CVDs such as coronary artery disease and mental disorders like depression. ${ }^{28}$ In PHC, the prevention of CVDs is a high priority. Thus, the relationship between depression and risk factors of CVDs such as hypertension is of great relevance for the public health sector. According to a meta-analysis of 41 cross-sectional studies ${ }^{29}$ the prevalence of depression in patients with hypertension was much higher than in the general population (26.8\% compared with $4.4 \%$ ), suggesting that the two are strongly connected.

\section{Potential mechanisms linking depression and hypertension}

Some mechanisms have been proposed to explain how depression is linked to hypertension. First, people living with depression tend to have unhealthy lifestyles which include habits such as smoking, alcohol abuse and physical inactivity, ${ }^{30}$ all of which are risk factors for hypertension and CVDs. Second, depression can cause autonomic nervous system dysfunctions which activates sympathetic activities $^{31}$ thereby elevating blood pressure. Insomnia and short sleep duration, which are typical symptoms of some forms of depression, have been found to significantly increase the risk of hypertension incidence. ${ }^{32} 33$ Little sleep can activate the hypothalamic-pituitary-adrenal axis, which raises blood pressure in the short term, and can lead to long-term structural adaptation that gradually reset the cardiovascular system to operate at an elevated pressure equilibrium. Finally, beyond its role in the aetiology of hypertension and CVDs, the presence of depression may also affect the treatment of hypertension. It was found that physicians were more cautious with augmenting antihypertensive treatment in people with depression ${ }^{34}$ because some antihypertensive medications have been found to cause or worsen depression. ${ }^{35}$ This means that depressed persons may be less likely to receive adequate treatment from their physician for their blood pressure. In another sense, depression is a risk factor for poor adherence to antihypertensive medication. ${ }^{36}$

The literature thus far explores the association of depression with three main facets of hypertension: the incidence of hypertension among those with previous normal blood pressure, the control of blood pressure among those with a previous diagnosis of hypertension and the course of blood pressure on a continuous scale.

\section{Evidence on depression and hypertension incidence}

The goal of primary prevention in PHC in terms of blood pressure is to prevent people from developing hypertension. According to a meta-analysis of longitudinal studies, depression significantly increased the risk of incident hypertension (RR 1.42; $95 \%$ CI 1.09 to 1.86 ).${ }^{37}$ However, 
authors cautioned that the limited number of longitudinal studies available may have impacted conclusions. It should also be noted that definitions of hypertension differed among studies, which included either high blood pressure measurement (with differing cut-offs such as $\geq 140 / 90 \mathrm{~mm} \mathrm{Hg}$ or $\geq 165 / 95 \mathrm{~mm} \mathrm{Hg}$ ), prescribed antihypertensive medication, physician diagnosis, self-reported hypertension or a combination of these. The inverse relationship (hypertension as a risk factor for incident depression) was assessed in another meta-analysis, which did not find a significant association. ${ }^{38}$ One possible explanation is that hypertension is often asymptomatic, having less impact on QoL and thus depression when compared with more advance stages of CVDs.

\section{Evidence on depression and hypertension control}

The goal of hypertension control in secondary prevention is for people with hypertension to reduce and maintain their blood pressure at a normal level through lifestyle changes and/or adhering to prescribed medication. Uncontrolled hypertension is the persistence of high blood pressure after a diagnosis of hypertension, which is a risk factor for developing CVDs. Despite being of great relevance for secondary and tertiary prevention in the public health sector, few studies have assessed the effect of depression on the control of hypertension among patients with hypertension. Depression was found to be positively associated with uncontrolled hypertension in a small cross-sectional study (RR 15.5; 95\% CI, not reported; $\mathrm{n}=40),{ }^{39}$ and a case-control study's adjusted model (RR 1.94; $95 \%$ CI 1.31 to $2.85 ; \mathrm{n}=590) .{ }^{40}$ In a large retrospective cohort study ( $\mathrm{n}=210482)$, the authors found a significant association between depression and uncontrolled hypertension in their secondary analysis (OR 1.21; 95\% CI 1.16 to 1.26$){ }^{41}$

\section{Evidence on depression and the course of blood pressure}

Looking at blood pressure on a continuous scale is also of interest to better understand the magnitude depression can impact blood pressure. One cross-sectional study $(n=2981)$ found that depressed subjects had lower mean systolic blood pressure than controls, and tricyclic antidepressant users had higher mean systolic and diastolic blood pressure. ${ }^{42}$ A recent longitudinal study in Germany $(\mathrm{n}=1887)$ also found that after 12 years of follow-up, a history of moderate major depressive disorder was associated with a decrease in both systolic and diastolic blood pressure. ${ }^{43}$ Since depressive symptoms vary over time, this study was limited by its definition of depression (a lifetime history of depression) because conclusions about the relative effect of short-term and long-term depressive symptoms could not be made. The importance of evaluating depressive symptoms in parallel to blood pressure over time was noted in another longitudinal study in Norway, ${ }^{44}$ which also found that baseline depression predicted lower blood pressure at year 22, but further found that a high symptom level of depression and anxiety at baseline and year 11 was more strongly associated with a decrease in blood pressure at year 22, and associated with an even stronger decrease in blood pressure if there were high levels of symptoms at all three examinations.

Other emotional states such as anxiety and stress have overlapping symptomology with depression but are distinct negative emotional states. The independent associations between anxiety and stress with hypertension have been studied.

\section{Evidence on anxiety and hypertension}

According to the Depression Anxiety Stress Scale (DASS), which differentiates the three emotional states, symptoms of anxiety include autonomic arousal (heart rate increase, mouth dryness, etc), skeletal muscle effects (trembling), feelings of panic, faintness or being terrified for no good reason. ${ }^{45}$ A meta-analysis which pooled 13 cross-sectional studies with 151389 subjects found a significant positive association between anxiety and hypertension (OR 1.40, 95\% CI 1.20 to 1.62$).{ }^{46}$ Although significant publication bias was detected, the OR remained significant after trim and fill analysis (OR 1.18, 95\% CI 1.02 to 1.37 ). In the same meta-analysis, eight prospective studies on baseline anxiety and incident hypertension were pooled $(n=80$ 146) and presented a HR by random effect model of 1.55 (95\% CI 1.24 to 1.94$)$ with strong heterogeneity $\left(\mathrm{p}<0.001, \mathrm{I}^{2}=84.6 \%\right)$ but no publication bias was detected $(\mathrm{p}=0.663)$. Although there are clear relationships, the mechanisms for them are not yet well understood.

\section{Evidence on stress and hypertension}

Symptoms of stress included in the DASS are difficulty relaxing, nervous arousal, getting easily upset or agitated, irritable/over-reactive and impatience. ${ }^{45}$ In a recent metaanalysis $(n=5696)$ of 11 studies, domains of mental stress were defined as psychological stress, anxiety/depression or work stress. ${ }^{47}$ Two studies $(n=622)$ looked at the association of mental stress on the risk of hypertension (OR $2.40,95 \%$ CI 1.65 to $3.49, \mathrm{I}^{2}=0 \%, \mathrm{p}=0.33$ ) and the other nine studies looked at the association of hypertension on the risk of mental stress ( $\mathrm{OR}=2.69,95 \%$ CI 2.32 to 3.11). The limitation of studies on stress and hypertension, as seen in the meta-analysis, are that they are few in numbers and have varying definitions of stress, which in some cases include depression and anxiety. Therefore, more studies are needed on the relationship between stress and hypertension, with a clear definition of stress as a distinct emotional state.

\section{Mental healthcare in Kosovo}

Supporting persons with mental illness in Kosovo is a challenge. This is because mental health services are only available on referral to a specialist, which may deter persons with mental illness from seeking care as it remains highly stigmatised in the country. Seeking professional support to address mental health problems is associated with 'tremendous shame' in the country; thus, support is rarely requested or is kept within the family circle. ${ }^{48}$ Indeed, only $15 \%$ of people who stated they needed help 
actually sought the help from a psychologist or psychiatrist due to fear of being stigmatised. ${ }^{11}$ If help for mental illness is sought outside the home, families often consult with traditional healers or local religious persons instead of mental health professionals. ${ }^{48}$

In summary, further research is needed to make sense of the inconsistencies in the literature between depression and the different facets of hypertension. Understanding potential mutual influences between depression and hypertension in Kosovo is highly relevant, as it could indicate the need for integrated mental health services in PHC, especially given that both depression and hypertension are common and standalone mental health services are stigmatised. Integrated mental health services have been found to be effective in another setting ${ }^{49}$ for more effective control of both depression and hypertension.

\section{Objectives of the KOSCO study}

The overarching goal of the 5-year KOSCO study is to contribute epidemiological evidence to the prevention and control of NCDs in Kosovo as the basis for policy and decision-making, which is currently lacking in the country. Specific objectives include:

1. To assess the prevalence and temporal change of NCDs such as hypertension, depression, diabetes and chronic obstructive pulmonary disease (COPD), as well as the prevalence and temporal change of aetiological risk factors, disease control and underdiagnosis of these NCDs among PHC users.

2. To evaluate the longitudinal relationship of PHC nonexperimental interventions such as motivational counselling sessions with adherence to healthy lifestyles (physical activity, nutrition, smoking, alcohol consumption), clinical measurements (blood pressure, BMI and HbAlc) and the stage of health behaviour change.

3. To assess the predictive association between depression and the course of blood pressure in adult PHC users living in Kosovo, as well as the mediators of the association.

\section{METHODS AND ANALYSIS \\ Study design}

This prospective 5-year longitudinal study of PHC users in Kosovo conducted follow-ups annually in two phases, spaced by approximately 6 months. Part 1 included an in-person interview and health examination, while part 2 included a telephone interview. Part 1 of baseline data collection began in March 2019 and part 2 began in October 2019. The second follow-up started in March 2020. Annual follow-ups during a 5-year cohort allows for potential mediation analysis.

\section{Setting}

The study was conducted in Kosovo, which is located in the centre of the Balkans and the newest independent state in Europe, although not accepted as such by all countries. It has a population of 1.8 million and is divided into
38 municipalities over a surface area of nearly $11000 \mathrm{~km}^{2}$. The country has mainly rural settlements $(62 \%)$, ethnicAlbanians with minorities of Serbian, Roma, Ashkali, Egyptian (RAE), Bosnians and Turkish ethnicities and has a male-to-female ratio of $1.06 .{ }^{5}$ Study sites included the 12 MFMCs from the following municipalities in Kosovo: Fushë Kosovë, Drenas, Gračanica, Gjakovë, Junik, Lipjan, Malishevë, Mitrovicë, Obiliq, Rahovec, Skënderaj and Vushtrri. There exists only one MFMC per municipality.

The study was embedded within the AQH project and the selection of municipalities was based on the project's established stakeholder collaboration. The AQH project engaged with these municipalities based on nine indicators: RAE population as percentage of total population, per capita public expenditure, per capita total PHC financing, social welfare beneficiaries per 100 inhabitants, female lone parent as percentage of female population, doctors per 1000 inhabitants, nurses per 1000 inhabitants, total PHC visits per capita, diarrhoea per 1000 inhabitants and applying a convenience sample to ensure geographical clustering and representation of ethnic-Serbs.

\section{Participants}

The study population included adults aged 40 years or older who consulted healthcare services at one of the 12 study sites on the day of recruitment. Persons were excluded from participating in the study if (1) they had a terminal illness, (2) were not able to understand or respond to prescreening questions, (3) did not live in 1 of the 12 study municipalities or (4) live abroad for more than 6 months of the year. Patients exiting the 12 participating MFMCs were approached consecutively and screened for inclusion and exclusion criteria. Informed consent was obtained from participants in a quiet room of the MFMC. Research nurses alternated municipalities in their study clusters each week of recruitment (Cluster 1: Gračanica, Drenas, Skënderaj. Cluster 2: Malishevë, Rahovec, Gjakovë, Junik. Cluster 3: Fushë Kosovë, Vushtrri, Mitrovicë. Cluster 4: Lipjan, Obiliq). Clusters were developed based on the proximity of municipalities to each other and the number of participants to be recruited per municipality to balance the workload of each research nurse.

\section{Incentive}

As a participant of the cohort, one was entitled to the following incentives: waived copayments of one health consultation and associated blood tests once per year, and an HbAlc test was free of charge to the participant on the day of each in-person interview.

\section{Study preparation}

Four research nurses were hired to conduct the data collection. They participated in a 3-day training certified by the Kosovo Nursing Chamber which covered standard operating procedures (SOP) to perform interviews and health assessments. One week prior to recruitment, research nurses and the field research coordinator visited 
all sites to meet and inform relevant staff about the study and ensure necessary on-site equipment was ready for use. A plan of the recruitment schedule, which rotated between study sites, was provided to all directors.

\section{Patient and public involvement}

Public stakeholder involvement in study design

Directors of the MFMCs were invited for meetings to discuss the study in October 2018 and in February 2019 ( 5 months and again 3 weeks prior to the launch of the recruitment of participants, respectively). In these meetings, the purpose and methods of the study were presented. Stakeholder feedback on logistical issues and health priorities in the regions were adapted into the protocol. For example, directors of PHC facilities asked to include data collection on respiratory health since their clinical experience indicated that it was a public health concern with lacking epidemiological evidence in the area. Considering the decentralised system, a signed agreement with all 12 directors of the MFMCs was established for their voluntary participation in the cohort.

Patient involvement in piloting the interview guide

The interview guide was piloted on a convenience sample of nine PHC patients from the MFMC in Obiliq. The questions were adapted according to patient feedback (eg, some questions were repetitive or not culturally appropriate, therefore removed). The first follow-up questionnaire was piloted on 42 cohort participants and the questionnaire was again modified based on feedback.

\section{Variables and data collection}

\section{Interviews}

The interview guide of the cohort addressed many objectives and was therefore lengthy. To reduce the risk of participant fatigue, the interview guide was divided into two parts, spaced by an interval of approximately 6 months. Part 1 of the interview was conducted in-person at the MFMC by a trained research nurse (approximately 30 min duration) and part 2 of the interview was conducted by telephone (approximately 20 min duration). Refer to table 1 for an overview of variables measured in each of the two parts of baseline data collection which are grouped by theme, and table 2 for a description of validated instruments used.

Data collection was divided into two parts: in-person interviews with health examination (part 1) and telephone interviews (part 2). Variables assessed are grouped by theme, and inclusion in part 1 and/or 2 is indicated with an ' $\mathrm{x}$ ' or with comments.

Table 1 Overview of variables measured in participant interviews and health examinations

\begin{tabular}{|c|c|c|c|}
\hline Theme & Variables & $\begin{array}{l}\text { Part 1: in-person } \\
\text { interview }\end{array}$ & $\begin{array}{l}\text { Part 2: telephone } \\
\text { interview }\end{array}$ \\
\hline $\begin{array}{l}\text { Sociodemographic } \\
\text { factors }\end{array}$ & $\begin{array}{l}\text { Age, gender, marital status, residence, ethnicity, } \\
\text { education level, occupation, household } \\
\text { composition, income level, pension, health } \\
\text { insurance }\end{array}$ & $x$ & \\
\hline $\begin{array}{l}\text { Social and environmental } \\
\text { factors }\end{array}$ & Social support, proximity to health services & $x$ & \\
\hline Health factors, block II & Somatic symptoms & & $x$ \\
\hline $\begin{array}{l}\text { Lifestyle behaviours, } \\
\text { block II }\end{array}$ & $\begin{array}{l}\text { Health behaviours and stages of change, Health } \\
\text { specific self-efficacy }\end{array}$ & & $x$ \\
\hline $\begin{array}{l}\text { Psychological factors, } \\
\text { block I }\end{array}$ & $\begin{array}{l}\text { Depression, anxiety, stress, resilience, post- } \\
\text { traumatic stress disorder, quality of life }\end{array}$ & $x$ & $\begin{array}{l}\text { Repeat only: depression, } \\
\text { anxiety, stress, quality } \\
\text { of life. Add: previous } \\
\text { diagnosis of mental illness }\end{array}$ \\
\hline $\begin{array}{l}\text { Psychological factors, } \\
\text { block II }\end{array}$ & General self-esteem & & $\mathrm{x}$ \\
\hline Health examination & $\begin{array}{l}\text { Blood pressure, height, weight, waist/hip/neck } \\
\text { circumferences, HbA1c, peak expiratory flow }\end{array}$ & $x$ & \\
\hline
\end{tabular}

CVD, cardiovascular diseases. 


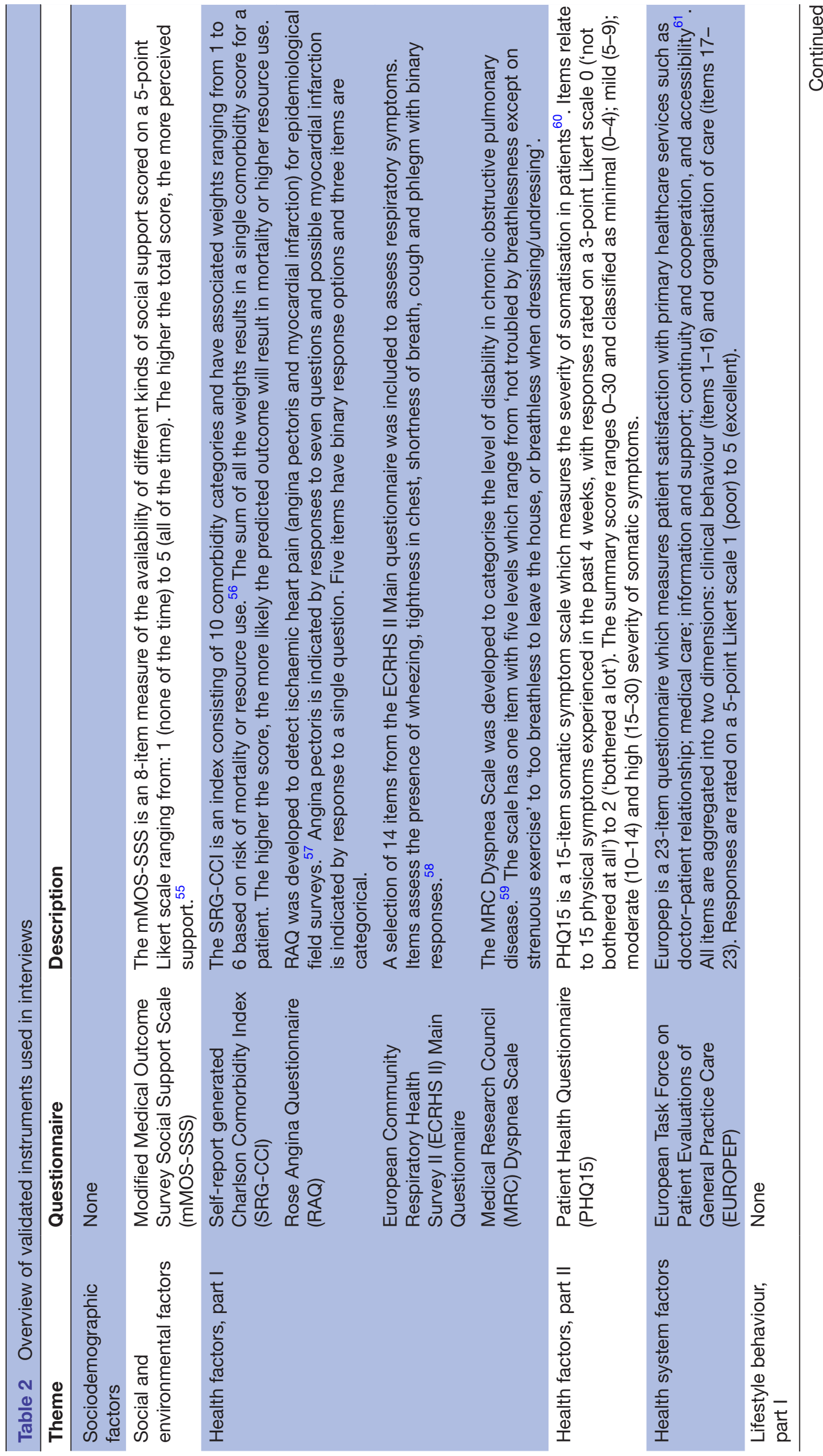




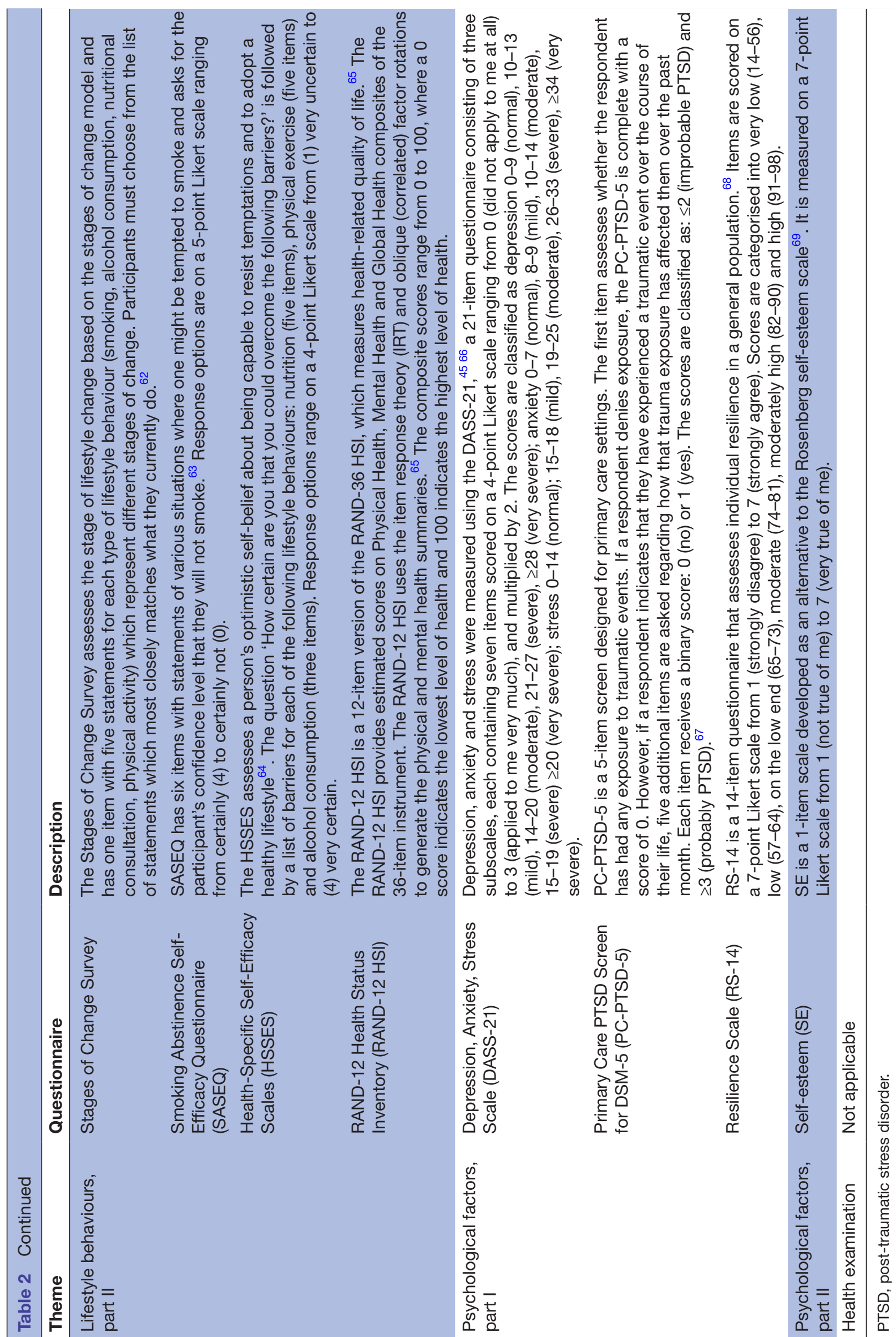


Validated instruments used in each of the interview themes are described in the table. Questions developed by the study team and questions from non-validated questionnaires are not included in the table.

\section{Physical examination}

Immediately following the in-person interview, the research nurse performed a brief health examination of about $10 \mathrm{~min}$.

- Height $(\mathrm{cm})$ and weight $(\mathrm{kg})$ were measured using stadiometers and scales which were available at the MFMCs (various brands). The precision of scales was assessed regularly with a weight of $10 \mathrm{~kg}$. Circumferences of the waist, hip and neck were measured using the SECA 201 measuring tape (Seca GmbH \& Co. KG., Switzerland).

- Peak expiratory flow (PEF) (L/min) was measured 3 times with $30 \mathrm{~s}$ pause between attempts, using the OMRON Peak Flow Meter PFM20 (Omron Healthcare, Switzerland). PEF predicted (\%) was calculated as follows: estimated (measured) PEF/expected PEF. The expected PEF values were derived based on age, gender and height using the regression equation developed by Hankinson et al. ${ }^{50}$

- Systolic and diastolic blood pressure (in $\mathrm{mm} \mathrm{Hg}$ ) were measured three times, at least $3 \mathrm{~min}$ apart, after sitting quietly for about $10 \mathrm{~min}$, using an M3 model Omron blood pressure monitor (Omron Healthcare, Switzerland). The research nurses placed the blood pressure cuff $2 \mathrm{~cm}$ above the elbow on the bare left upper arm (in the case of arteriovenous fistula, radiotherapy or removal of lymph nodes in the armpit of the left arm, the right arm was used) of the seated participant and elevated the arm on the table to the level of the fourth intercostal space.

- Towards the end of the battery of tests, the research nurse accompanied participants to the laboratory of the MFMC for a finger-prick (non-invasive) glycated haemoglobin test (HbA1c, \%). The HbA1c test was performed by an MFMC staffed laboratory technician who received training by the supplier on how to use the SUPER ID clinchem device (Dr. Müller Gerätebau GmbH, Germany).

- Participants were given a 'self-care passport' at baseline, which was developed by local experts in collaboration with the AQH project. The research nurses transcribed the participants' health examination results in the passport which also had additional space for participants to write blood pressure or blood glucose measurements taken at home. Participants were instructed that they will be recontacted in 6 months for a telephone interview.

\section{Definitions of main variables by objective} Objective 1

- Depression was defined as self-reported depression diagnosis by a healthcare professional and/ or prescribed antidepressant medications and/or a DASS depression score of $>13$ (moderate to very severe depressive symptoms). Uncontrolled depression was defined as being diagnosed with depression and/ or taking antidepressant medication yet having a DASS depression score of $>13$. Undiagnosed depression was defined as not being diagnosed with depression nor taking antidepressant medication yet having a DASS depression score of $>13$

- Hypertension was defined as a self-reported hypertension diagnosis by a healthcare professional and/or prescribed antihypertensive medications and/or a blood pressure measurement $\geq 140 / 90 \mathrm{~mm} \mathrm{Hg}$. Uncontrolled hypertension was defined as being diagnosed with hypertension and/or taking antihypertensive medication yet having a blood pressure measurement $\geq 140 / 90 \mathrm{~mm} \mathrm{Hg}$. Undiagnosed hypertension was defined as not being diagnosed with hypertension nor taking antihypertensive medication yet having a blood pressure measurement $\geq 140 / 90 \mathrm{~mm} \mathrm{Hg}$.

- Diabetes was defined as a self-reported diabetes diagnosis by a healthcare professional and/or prescribed antidiabetic medications and/or an HbAlc measurement $\geq 6.5 \%$. Uncontrolled diabetes was defined as being diagnosed with diabetes and/or taking antidiabetic medication yet having an HbA1c measurement $\geq 6.5 \%$. Undiagnosed diabetes was defined as not being diagnosed with diabetes nor taking antidiabetic medication yet having an $\mathrm{HbAlc}$ measurement $\geq 6.5 \%$.

- $C O P D$ was defined as a self-reported COPD diagnosis by a healthcare professional and/or a PEF $<80 \%$ predicted with breathlessness and/or cough symptoms for greater than 6 months. Uncontrolled COPD was defined as being diagnosed with COPD yet having a $\mathrm{PEF}<80 \%$ predicted. Undiagnosed COPD was defined as not being diagnosed with COPD yet having a PEF $<80 \%$ predicted with breathlessness and/or cough symptoms for greater than 6 months. A PEF $<80 \%$ predicted with respiratory symptoms (breathlessness or cough for greater than 6 months) was found to be an appropriate cut-off to detect COPD in the absence of spirometry. ${ }^{51}$

- Lifestyle factors included: smoking (current smoker, ex-smoker, never smoker), meeting WHO recommendations for physical activity (at least $150 \mathrm{~min}$ of moderate-intensity physical activity throughout the week, or at least 75 min of vigorous-intensity physical activity throughout the week, or an equivalent combination of moderate-intensity and vigorous-intensity activity $^{52}$ ), meeting WHO recommendations for fruit and vegetable intake (at least five portions (400 g) of fruits and vegetables per day $\left.{ }^{53}\right)$, binge drinking (consumption of $\geq 60 \mathrm{~g}$ of pure alcohol (six or more standard drinks) on at least one single occasion at least once in a month ${ }^{54}$ ), and BMI: weight $(\mathrm{kg}) /$ height $\left(\mathrm{m}^{2}\right)$. 


\section{Objective 2}

- Motivational counselling sessions: are a nonexperimental community intervention, where all patients being treated at a MFMC, FMC or FMA aged 40 or older with diabetes, hypertension or at risk for developing diabetes and/or hypertension are eligible to be referred by a doctor or nurse to the nearest health resource centre. There, the nurse provides one-on-one motivational counselling sessions on lifestyle changes based on the patient's needs. Prior to rolling out this intervention, several preparatory steps were undertaken by the AQH project. First, health resource centres were established within MFMCs as a new location for nurses to provide motivational counselling sessions and other preventive services for management of NCDs. Furthermore, nurses from MFMCs completed several training sessions on motivational counselling. At the time of baseline, motivational counselling was offered in 5 of the 12 study sites (Fushë Kosovë, Gjakovë, Malishevë, Mitrovicë and Vushtrri), and a staggered introduction of the intervention to other study sites is anticipated. Attending a motivational counselling session was the main exposure, a dichotomous variable where participants answered yes or no to the question: 'Have you ever participated in a motivational counselling session/health education session with a nurse in a health resource centre?'

- Lifestyle factors were among the main outcomes, described under 'objective 1'.

- Clinical measurements were among the main outcomes, which were continuous variables that included blood pressure, BMI and HbA1c described under 'physical examination'.

- Stage of behavioural change was one of the main outcomes, an ordinal variable assessed using the Stages of Change Survey. For each lifestyle (smoking, nutrition, physical activity and alcohol consumption), participants were categorised into one of the following stages of change based on their responses: maintenance, action, preparation, contemplation and precontemplation.

- Patient satisfaction and quality of care were predictors included in the secondary analysis. Patient satisfaction was a binary variable defined as an average EUROPEP score per item of $\geq 4$. Quality of care was a continuous variable defined as the number of patient-reported healthcare provider actions completed from the list of recommendations in the PEN protocol during the participant's last visit in a PHC centre.

\section{Objective 3}

- Depression was the main exposure, a dichotomous variable defined under 'objective 1'.

- Change in blood pressure was the main outcome, which was the blood pressure (described under "physical examination') from baseline subtracted from blood pressure at follow-up.
- Hypertension incidence, uncontrolled hypertension and underdiagnosed hypertension are secondary binary outcomes described under 'objective 1'.

\section{Non-participants}

Non-participants (patients approached who declined to participate or who did not meet inclusion criteria) were asked nine optional questions with the purpose to understand if participants differ from non-participants. The optional questions provided information on sex, age, education level, diagnosis of diabetes, lung disease, CVD, smoking status, weight, level of satisfaction with PHC services and reason for non-participation.

\section{Data management}

Data from in-person and telephone interviews were collected using Open Data Kit (ODK) software. Results from health examinations were also entered into ODK. Data quality was assured through (1) formulation of SOPs for all aspects of the study, (2) extensive and careful training of the study team according to the SOPs, (3) onsite supervision of field activities ensuring adherence to protocol and (4) regular monitoring and internal evaluation of data entry during the field visits. The ODK and STATA programmes kept track of all changes made to the data. All data were merged into a single database at the end of data entry using STATA V.15.1 (STATA Corporation).

\section{Power calculation}

Power calculation without local effects

The following is a power calculation for the longitudinal study of the association of change in blood pressure with depression in the case of a single homogenous population with the prevalence of depression $d=40 \%$. We denote the relative effect of the depression at baseline on the change of blood pressure at follow-up as tau. For a small effect tau=0.25, which under the normal distribution assumption corresponds to the shift from the median to the 60 percentile, and assuming a $20 \%$ loss to follow-up, we arrive at the minimal cohort size of 883 people for $90 \%$ power. The control for confounding variables will lead to a reduction of power, as will the discretisation of the blood pressure measurement to study hypertension as a binary outcome, and so we aim to recruit a total of 1000 patients into the cohort. The number of participants to be recruited by each MFMC was proportional to their mean number of medical visits in the months of June 2018 and October 2018.

\section{Power in the presence of clustering}

To take into account the potential local variation in the effect of depression on blood pressure, we performed explicit simulations to make sure that the study has sufficient power under a range of plausible scenarios. Specifically, we posited that the mean blood pressure can vary between the 12 municipalities (random effect with variance sigma 2 ), and also that the effect of depression on blood pressure can be different in each municipality 
Table 3 Simulation of statistical power

\begin{tabular}{lllllll} 
& Tau=0.25 & Tau=0.30 & Tau=0.35 & Tau=0.4 & Tau=0.45 & Tau=0.5 \\
\hline Rho=0.1 & $80 \%$ & $91 \%$ & $97 \%$ & $99 \%$ & $99 \%$ & $99 \%$ \\
Rho=0.2 & $66 \%$ & $80 \%$ & $91 \%$ & $96 \%$ & $98 \%$ & $99 \%$ \\
Rho=0.3 & $49 \%$ & $65 \%$ & $77 \%$ & $87 \%$ & $93 \%$ & $97 \%$ \\
\hline
\end{tabular}

(random effect with variance rho 2). The magnitudes sigma and rho of these local effects were tunable parameters of the simulation, as was the overall effect size tau. Preliminary analyses showed that the power of the study is driven by the relationship of tau and rho, and is not sensitive to sigma; this is because the municipality-level effect affects depressed and non-depressed people equally. Thus, we fixed sigma=tau in what follows.

For 18 combinations of plausible values of tau and rho, we simulated normal data on 800 participants (ie, the target cohort size minus $20 \%$ loss to follow-up) 10000 times and computed the fraction of instances when the mixed regression model fitted on this synthetic data reported depression as a significant factor. This fraction can be interpreted as the statistical power of the study for the given tau and rho. The results of the simulations are reported in table 3 (rounded down to the nearest percent). We found that the study retains sufficient power for as long as the overall effect of depression dominates the local variation in that effect (that is tau is much greater than $r h o$ ), which is likely. This requirement is progressively relaxed as the overall effect size grows.

The simulations did not take into account the loss of power due to adjustment for confounders.

\section{Statistical analysis plan}

Figure 1 provides an overview of associations of interest for the cohort. Statistical methods are presented by objective.

\section{Objective 1}

The descriptive statistics of depression, hypertension, diabetes, COPD as well as the control and underdiagnoses of these diseases will be presented as follows: categorical variables will be presented as numbers and percentages. Normally distributed quantitative variables will be presented as mean and SD. Other quantitative variables will be presented as medians and IQRs. $\chi^{2}$ tests, t-tests and Wilcoxon rank-sum tests will be used for bivariate analysis where appropriate, such as to assess differences by age, gender or socioeconomic status (SES).

\section{Objective 2}

The main exposure of interest was attendance in motivational counselling sessions (non-experimental PHC intervention). In light of the absence of pure controls for this non-experimental intervention, comparisons will be made between those who chose to participate in the invention and those who did not within the same centre. The first outcome variable was stage of behaviour change, which was ordinal (maintenance, action, preparation, contemplation and precontemplation). Two approaches will be used: initially, the analysis will be done using ordinal regression, then the outcome variable will be split into two categories: (1) high motivation (maintenance, action, preparation) and (b) low motivation (contemplation and precontemplation) and logistic regression will be applied. The second outcome was adherence to the following aspects of healthy lifestyles: nutrition, physical activity, alcohol consumption and smoking. Logistic regression analysis will be conducted for each lifestyle. For the third outcome (clinical measurements), a mixed linear regression model will be constructed for each outcome of interest: blood pressure, BMI and HbA1c. Comorbidities, physical ability, SES, living status and employment status are potential confounders and will be controlled for during data analysis. The intrinsic municipality and participant effects will be modelled with random effects. Potential effect modifiers include sex, age and social support. Self-efficacy will be considered as a potential mediator. Secondary analyses with predictors of patient satisfaction and quality of care will be conducted with logistic regression models.

\section{Objective 3}

The main outcome for this objective was change in blood pressure. Secondary outcomes included hypertension incidence, control and underdiagnosis. We will use an explanatory model with a focus on depression among predictor variables. Covariates systematically considered as confounders as well as effect modifiers in all models will be sex, age, urban/rural, ethnicity, education level and employment status. Additional covariates considered in some models include: smoking, alcohol, physical activity, obesity, family history, anxiety, stress, PTSD, resilience, social support, self-esteem, health literacy, healthcare seeking, patient satisfaction, comorbidity, sleep quality and duration, and medication. Antidepressant use and lifestyle factors will be assessed as a potential mediators.

Two approaches will be explored to assess the longitudinal association between depression and change in blood pressure.

1. Predictive perspective: a regression model will be constructed with the outcome of change in blood pressure from baseline (continuous). Baseline blood pressure will be adjusted for by including it as a covariate in the model. This model will allow predicting the future course of blood pressure, based on a set of variables observed at baseline. This model is of value for a provider 


\section{Socio-demographic factors} (age, gender, marital status, residence, ethnicity, education level, occupation, household composition, income level, pension, health insurance)

Social and Environmental factors
(Social support, proximity to health
services)

\section{Health factors}

(comorbidities; disabilities; symptoms; family history; somatic symptoms; sleep)
Quality of life

Incidence, control, underdiagnosis of NCDs

Blood pressure

Glycated

haemoglobin

Peak Expiratory Flow

Disease complications

Health service use

Change in lifestyle

Physical activity Smoking Alcohol Diet BMI

\section{Personal factors}

(resilience; self-esteem; post-traumatic stress disorders; depression; anxiety; stress)
Stage of behavioural change

Figure 1 Hypothesised associations between variables under study. The hypothesised associations between outcome variables on the right, predictor variables on the left and mediating variables in the middle are represented in the figure. Sociodemographic factors, social and environmental factors, health literacy and self-care, as well as health system factors are thought to impact the outcome of quality of life, the incidence and control of chronic diseases and lifestyle change, and mediated by personal and health factors. BMI, body mass index; NCD, non-communicable disease.

perspective: based on what the provider observes at a specific point in time, what is the predicted course of blood pressure?

2. Change perspective: the effect of change in depression (predictor) on change in blood pressure (outcome) will be assessed with a repeated measures model. This model will allow assessing the parallel change in depression and blood pressure and in that sense takes cross-sectional short-term associations at baseline and follow-up into consideration.

Analyses with secondary outcomes of hypertension incidence, control and underdiagnosis will be conducted with logistic regression models. The secondary outcomes are relevant for primary and secondary prevention in PHC. Anxiety and stress will also be included as focal predictor variables in secondary analyses.

\section{Strengths and limitations}

Given the limited evidence on NCDs in Kosovo, the cohort is of great benefit for healthcare decision-makers which rely on health data. Results from this cohort study will provide an overall insight into the relationship between NCDs and their determinants through study objective 1. Considering that this study is assessing the longitudinal association of PHC interventions (such as delivery of motivational counselling sessions for behaviour change) in study objective 2, the scientific findings of this study can be applied in designing targeted behaviour change interventions. Behaviours affect morbidity, and extremely unhealthy behaviours may lead to mortality, therefore understanding what causes patients to do certain behaviours and what motivates them to change, provides information which could be useful for populations with similar characteristics. ${ }^{14}$ Further, understanding potential mutual influences between depression and hypertension could indicate the need for integrated mental health services in PHC for more effective control of both conditions ${ }^{46}$ and will be addressed through objective 3 . 
Having embedded this cohort in an existing local implementation project, namely AQH which builds on strong partnerships with local stakeholders, greatly increased the ease of implementation and acceptability of this study. For example, the study population lives in mostly rural areas, with high levels of poverty and low levels of education which meant that there was little awareness of research and their benefits. Being embedded within the AQH project, which had established trust with municipalities, helped in the recruitment process. Further, given that the healthcare system is decentralised, getting directors of MFMCs from multiple municipalities on board to participate in the cohort study would normally be a long and complicated process, but was simplified since the directors had a longstanding relationship with the AQH project.

A pilot of the questionnaire with 9 PHC users aged 40 years and above conducted in March 2019 and in the MFMC of Obiliq and again in October 2019 with 42 cohort participants from various municipalities served to identify the understanding and flow of questions. Some questions were identified as inappropriate or irrelevant in the cultural context and were omitted. For example, the original PC-PTSD-5 questionnaire listed sexual abuse as an example of a traumatic event and this was considered offensive by one person in the pilot survey. Thus, the example was removed. One question asked if the participant had ever been diagnosed with a mental disorder by a physician. Local research nurses unanimously stated that this question was not culturally acceptable since it was not perceived well by the participants; therefore, it was removed from part 1 of the baseline data collection (initial contact with participants) and moved to part 2 to allow participants time to grow trust with research nurses. The PHQ asks questions about menstruation pain during intercourse, which were also culturally unacceptable and considered unpleasant to ask in an interview and a disclaimer statement was added before the items to preface the question. A question in the EUROPEP which assesses satisfaction with 'getting through to the practice on telephone' was removed as it is not common practice in Kosovo. Given that the average level of education in Kosovo's older population is of primary school or lower, some instruments' questions were abstract and difficult to understand by participants. In particular, respondents of the pilot survey noted that multiple questions in the RS-14 were difficult to understand, such as 'I am friends with myself' which was considered a Western ideal and 'I keep interested in things' often yielded participant questions like 'what things?' A debriefing with research nurses ensured that these questions were clarified with participants in a uniform way. It was confirmed through the pilot that the questionnaire was too lengthy, demonstrated by participants asking to end the interview before the end. It was decided to separate questions into two parts, asked with an interval of 6 months.

During the preparation period which involved site visits before the launch of recruitment, the first author learnt that lab hours were shorter than anticipated, which limited the amount of hours of recruitment (from 7:00 to 13:00). This meant that the original estimated recruitment timeline was extended from 3 months to 8 months. Further, participation rates were low, which extended recruitment time.

Due to the recruitment scheme in PHC facilities, the study is not population based. Thus, the study is limited in its generalisability as well as it may overestimate the prevalence of health conditions. However, patients visit MFMCs for an array of conditions as well as for general check-ups; thus, healthy persons are also included in the study. Providing participants incentives with free health consultations may bias towards participation of persons with chronic conditions, and thus may also overestimate the prevalence of NCDs and their determinants. The relevance of the study, in the absence of being entirely representative for Kosovo as a whole, lies in the longitudinal design; furthermore, it evaluates care and its perception and utilisation in a large number of relevant health service infrastructures. The findings will therefore be relevant and guiding for other similar structures in the country.

The non-randomised nature of PHC interventions mentioned in this study is a limitation in the interpretation of results regarding the effectiveness of interventions. But randomisation of interventions was not possible: centres offering the interventions were selected based on feasibility and interest of the MFMC staff to increase success of the pilot health centres, and the intervention is offered to all patients; therefore, exposure is self-selected.

\section{ETHICS AND DISSEMINATION}

\section{Ethics approval and consent to participate}

Ethical approvals for the study were obtained from Ethics Committee Northwest and Central Switzerland (reference number 2018-00994) on 11 December 2018 and the Kosovo Doctors Chamber (reference number 11/2019) obtained on 30 January 2019 and expiring on 31 December 2023. Before any data were collected, participants were asked for their verbal and written consent. To obtain consent, participants were informed that (1) their participation was voluntary, (2) they could withdraw from participation at any time and (3) non-participation would not have any negative effects. The participants were asked for additional consent whether, in the case a previously unrecognised medical problem was detected, they approve that qualified staff or the research team would inform them of the results and provide advice on what the participant should do next. SOP developed by the study team and approved by MFMC directors (who are physicians), were provided to research nurses to guide them in referring participants to appropriate care. Severe findings (systolic blood pressure $\geq 180 \mathrm{~mm} \mathrm{Hg}$ and diastolic blood pressure $\geq 110$ ) were referred immediately to emergency services in the MFMC. Participants were informed how the data will be used and that confidentiality is ensured as their 
data are coded. Potential risks and benefits of participation were also discussed with participants, and ample time was given to ask questions. Once consent was obtained, the research nurse proceeded to data collection.

\section{Data protection}

Data entry was done using a tablet (Samsung Galaxy Tab A, Samsung Group, Switzerland), where data were sent to a server and erased from tablets daily. Only participant identifiers, but not names of the participants were included in electronic health databases. HbAlc results were recorded in laboratories as per facility protocol with participant name, but not participant ID. Consent forms were kept in a locked file cabinet in Pristina, with restricted access to project personnel. Each participant has a code which is linked to their personal identifying data (PID) and a code linked to the study data (DID). The participant identifying information with PID is kept in one document stored by the Deputy Team Leader of the AQH project in Pristina, Kosovo. The DID, study data and key which links PID and DID are kept in a passwordprotected document with the principal investigator $(\mathrm{NPH})$ in Basel, Switzerland.

\section{Collaboration}

The overall coordination of the cohort activities is the joint responsibility of the Board of Collaboration, which consists of two representatives from the University of Prishtina, two representatives of Swiss TPH and two representatives from National Institute of Public Health. Focus, content and protocols for follow-up assessments of the KOSCO study are approved by the Board of Collaboration.

Research questions assessed using cohort data will be published in scientific journals.

\section{Author affiliations}

${ }^{1}$ Epidemiology and Public Health, Swiss Tropical and Public Health Institute, Basel, Switzerland

${ }^{2}$ University of Basel, Basel, Switzerland

${ }^{3}$ Swiss Centre for International Health, Swiss Tropical and Public Health Institute, Basel, Switzerland

${ }^{4}$ National Institute of Public Health, Prishtina, Kosovo

${ }^{5}$ University of Prishtina, Prishtina, Kosovo

${ }^{6}$ Accessible Quality Healthcare Project, Prishtina, Kosovo

${ }^{7}$ University Clinical Centre of Kosovo, Prishtina, Kosovo

Acknowledgements The implementation of the cohort study protocol and prioritisation of study objectives would not be possible without the support of the Project Implementation Unit of the AQH project. Staff from 12 participating Main Family Medicine Centers (MFMCs) supported the study along with MFMC directors: Osman Maxhera (Fushë Kosovë), Kujtim Zarari (Gjakovë), Ismet Bogiqi (Drenas), Mirjana Dimitrijević (Gračanica), Ali Kuqi (Junik), Agim Krasniqi (Lipjan), Nuhi Morina (Malishevë), Fevzi Sylejmani (Mitrovicë), Haki Jashari (Obiliq), Jusuf Korenica (Rahovec), Fazli Kadriu (Skenderaj), Luljeta Zahiti-Preteni (Vushtrri). The KOSCO research nurses were instrumental in the success of recruitment and data collection: Tevide Bllaca, Arizona Igrishta, Selvete Zyberaj, Shqipe Agushi and Alma Stojanović.

Contributors KAO: codeveloped and implemented the study protocol, coordinated and supervised data collection, will analyse and interpret data. NJ contributed to study objectives related to non-communicable diseases in Kosovo. SS contributed to study objectives related to mental health in Kosovo. MK conducted statistical power calculations and will supervise data analysis. MZ, QR, AB-K and JG contributed to the study objectives related to the evaluation of health service provision and to the integration of the study protocol within the AQH framework. $\mathrm{NP}-\mathrm{H}$ developed the KOSCO cohort concept, study objectives and protocol, directed the implementation, data analysis and result interpretation. All authors read and approved the final protocol.

Funding This work was supported by the Swiss Agency for Development and Cooperation (SDC). The first year of salary for the doctoral studies of KAO and the implementation and running costs of the cohort were funded by SDC, which is an agency in the federal administration of Switzerland and part of the Federal Department of Foreign Affairs, whom are responsible for coordinating Swiss international development projects in Eastern Europe. They are the core funders of the AQH implementation project in which the cohort is embedded. Local SDC representatives were responsible for approving the cohort budget and the study proposal. SDC contributed to the direction of study objectives. The Swiss Tropical and Public Health Institute (Swiss TPH) has internally funded the salary of KAO in the second and third years of the doctoral studies. Coauthors associated with Swiss TPH include KAO, JG, ABK, MZ and NPH and contributed to the study in various capacities, specified in authors' contributions declaration. The Swiss Government Excellence Scholarship for Foreign Scholars and Artists was awarded to ABK for the time period of 2019-2022 (Reference number 2019.0234), which will fund her doctoral studies salary.

Competing interests $\mathrm{KAO}, \mathrm{ABK}$ and $\mathrm{QR}$ report personal fees from Swiss Agency for Development and Cooperation (SDC) during the conduct of the study. ABK reports grants from the Swiss Confederation during the conduct of the study.

Patient and public involvement Patients and/or the public were involved in the design, or conduct, or reporting, or dissemination plans of this research. Refer to the Methods section for further details.

Patient consent for publication Not required.

Ethics approval Ethical approvals for the study were obtained from Ethics Committee Northwest and Central Switzerland (reference number 2018-00994) on 11 December 2018 and the Kosovo Doctors Chamber (reference number 11/2019) obtained on 30 January 2019 and expiring on 31 December 2023.

Provenance and peer review Not commissioned; externally peer reviewed.

Open access This is an open access article distributed in accordance with the Creative Commons Attribution Non Commercial (CC BY-NC 4.0) license, which permits others to distribute, remix, adapt, build upon this work non-commercially, and license their derivative works on different terms, provided the original work is properly cited, appropriate credit is given, any changes made indicated, and the use is non-commercial. See: http://creativecommons.org/licenses/by-nc/4.0/.

ORCID iD

Katrina Ann Obas http://orcid.org/0000-0001-9110-5331

\section{REFERENCES}

1 World Bank. Life expectancy at birth, total (years), 2017. Available: https://data.worldbank.org/indicator/SP.DYN.LEO0.IN?locations=XKRS-AL-MK-ME [Accessed 22 Sep 2019].

2 Ahmeti M, Gashi M, Sadiku L, et al. Efficiency and effectiveness in implemention of unified and integrated health information system 2017;50.

3 Jerliu N, Toçi E, Burazeri G, et al. Prevalence and socioeconomic correlates of chronic morbidity among elderly people in Kosovo: a population-based survey. BMC Geriatr 2013;13:22.

4 Institute of Health Metrics and Evaluation. GBD results tool, 2016. Available: http://ghdx.healthdata.org/gbd-results-tool [Accessed 25 Jul 2018].

5 Kosovo Agency of Statistics. Population by gender, ethnicity at settlement level, 2013. Available: http://ask.rks-gov.net/media/1614/ population-by-gender-ethnicity-at-settlement-level.pdf [Accessed 30 May 2018].

6 Ferrari AJ, Charlson FJ, Norman RE, et al. Burden of depressive disorders by country, sex, age, and year: findings from the global burden of disease study 2010. PLoS Med 2013;10:e1001547.

7 Wenzel T, Rushiti F, Aghani F, et al. Suicidal ideation, post-traumatic stress and suicide statistics in Kosovo 2009;19:10.

8 Eytan A, Gex-Fabry M. Use of healthcare services 8 years after the war in Kosovo: role of post-traumatic stress disorder and depression. Eur J Public Health 2012;22:638-43.

9 Kashdan TB, Morina N, Priebe S. Post-Traumatic stress disorder, social anxiety disorder, and depression in survivors of the Kosovo 
war: experiential avoidance as a contributor to distress and quality of life. J Anxiety Disord 2009;23:185-96.

10 Nakayama R, Koyanagi A, Stickley A, et al. Social networks and mental health in post-conflict Mitrovica, Kosova. BMC Public Health 2014;14:1169.

11 Shkcmbi F, Melonashi E, Fanaj N. Personal and public attitudes regarding help-seeking for mental problems among Kosovo students. Procedia Soc Behav Sci 2015;205:151-6.

12 Ringdal Gl, Ringdal K. War experiences and general health among people in Bosnia-Herzegovina and Kosovo. J Trauma Stress 2016;29:49-55.

13 International Conference on primary health care. Declaration of Alma-Ata, 1978. Available: http://link.springer.com [Accessed $24 \mathrm{Jul}$ 2018].

14 Clark DW, MacMahon B. Preventive medicine. little, brown, 1967

15 World Health Organization. Package of essential noncommunicable (Pen) disease interventions for primary health care in low-resource settings, 2010. Available: https://www.who.int/nmh/publications/ essential_ncd_interventions_Ir_settings.pdf

16 Lianov L, Johnson M. Physician competencies for prescribing lifestyle medicine. JAMA 2010;304:202-3.

17 Somerville M, Kumaran K, Anderson R. Public health and epidemiology at a glance. Second edition. Chichester, West Sussex: Wiley Blackwell, 2012. https://www.wiley.com/en-us/Public+ Health+and+Epidemiology+at+a+Glance\%2C+2nd+Edition-p9781118999325

18 World Health Organization. Prevention of cardiovascular disease: guidelines for assessment and management risk of cardiovascular risk. Geneva: World Health Organization, 2007.

19 Arena R, Guazzi M, Lianov L, et al. Healthy lifestyle interventions to combat noncommunicable disease-a novel nonhierarchical connectivity model for key stakeholders: a policy statement from the American heart association, European Society of cardiology, European association for cardiovascular prevention and rehabilitation, and American College of preventive medicine. Eur Heart J 2015;36:2097-109.

20 Williams B, Mancia G, Spiering W, et al. 2018 ESC/ESH guidelines for the management of arterial hypertension: the task force for the management of arterial hypertension of the European Society of cardiology and the European Society of hypertension: the task force for the management of arterial hypertension of the European Society of cardiology and the European Society of hypertension. J Hypertens 2018;36:1953-2041.

21 Gorgieva GS, Stasević Z, Vasić S, et al. Screening of chronic diseases and chronic disease risk factors in two rural communities in Kosovo. Cent Eur J Public Health 2010;18:81-6.

22 Hashani V, Hyska J, Roshi E. Socioeconomic determinants of hypertension in the adult population of transitional Kosovo 2013;5

23 Markoglou NC, Hatzitolios Al, Savopoulos CG, et al. Epidemiologic characteristics of hypertension in the civilians of Kosovo after the war. Cent Eur J Public Health 2005;13:61-5.

24 Stasević Z, Gorgieva GS, Vasić S, et al. High prevalence of kidney disease in two rural communities in Kosovo and Metohia. Ren Fail 2010;32:541-6.

25 Tishukaj F, Shalaj I, Gjaka M, et al. Physical fitness and anthropometric characteristics among adolescents living in urban or rural areas of Kosovo. BMC Public Health 2017;17:711.

26 Gashi S, Berisha M, Ramadani N, et al. Smoking behaviors in Kosova: results of steps survey. Zdr Varst 2017;56:158-65.

27 Accessile Quality Healthcare Project. Knowledge, Attitudes, Practices, and Behaviour: Non-Communicable Diseases, Child Health and Citizens' Right to Health in Kosovo, 2016. Available: http://www.aqhproject.org/wp-content/uploads/2017/03/KAPBReport-2016 English.pdf [Accessed 9 Sep 2019].

28 De Hert M, Detraux J, Vancampfort D. The intriguing relationship between coronary heart disease and mental disorders. Dialogues Clin Neurosci 2018;20:31-40.

$29 \mathrm{Li}$ Z, Li Y, Chen L, et al. Prevalence of depression in patients with hypertension. Medicine 2015;94:e1317.

30 Bonnet F, Irving K, Terra J-L, et al. Anxiety and depression are associated with unhealthy lifestyle in patients at risk of cardiovascular disease. Atherosclerosis 2005;178:339-44.

31 Koschke M, Boettger MK, Schulz S, et al. Autonomy of autonomic dysfunction in major depression. Psychosom Med 2009;71:852-60.

32 Fernandez-Mendoza J, Vgontzas AN, Liao D, et al. Insomnia with objective short sleep duration and incident hypertension: the Penn state cohort. Hypertension 2012;60:929-35.

33 Gangwisch JE, Malaspina D, Posner K, et al. Insomnia and sleep duration as mediators of the relationship between depression and hypertension incidence. Am J Hypertens 2010;23:62-9.
34 Moise N, Davidson KW, Chaplin W, et al. Depression and clinical inertia in patients with uncontrolled hypertension. JAMA Intern Med 2014;174:818-9.

35 Celano CM, Freudenreich O, Fernandez-Robles C, et al. Depressogenic effects of medications: a review. Dialogues Clin Neurosci 2011;13:109-25.

36 Eze-Nliam CM, Thombs BD, Lima BB, et al. The association of depression with adherence to antihypertensive medications: a systematic review. J Hypertens 2010;28:1785-95.

37 Meng L, Chen D, Yang Y, et al. Depression increases the risk of hypertension incidence: a meta-analysis of prospective cohort studies. J Hypertens 2012;30:842-51.

38 Long J, Duan G, Tian W, et al. Hypertension and risk of depression in the elderly: a meta-analysis of prospective cohort studies. $J$ Hum Hypertens 2015;29:478-82.

39 Rubio-Guerra AF, Rodriguez-Lopez L, Vargas-Ayala G, et al. Depression increases the risk for uncontrolled hypertension. Exp Clin Cardiol 2013;18:10-12.

40 Almas A, Patel J, Ghori U, et al. Depression is linked to uncontrolled hypertension: a case-control study from Karachi, Pakistan. J Ment Health 2014;23:292-6.

41 Elperin DT, Pelter MA, Deamer RL, et al. A large cohort study evaluating risk factors associated with uncontrolled hypertension. $J$ Clin Hypertens 2014;16:149-54.

42 Licht CMM, de Geus EJC, Seldenrijk A, et al. Depression is associated with decreased blood pressure, but antidepressant use increases the risk for hypertension. Hypertension 2009;53:631-8.

43 Speerforck S, Dodoo-Schittko F, Brandstetter S, et al. 12-Year changes in cardiovascular risk factors in people with major depressive or bipolar disorder: a prospective cohort analysis in Germany. Eur Arch Psychiatry Clin Neurosci 2019;269:565-76.

44 Hildrum B, Romild U, Holmen J. Anxiety and depression lowers blood pressure: 22-year follow-up of the population based HUNT study, Norway. BMC Public Health 2011;11:601.

45 Lovibond PF, Lovibond SH. The structure of negative emotional states: comparison of the depression anxiety stress scales (DASS) with the Beck depression and anxiety inventories. Behav Res Ther 1995;33:335-43.

46 Pan Y, Cai W, Cheng Q, et al. Association between anxiety and hypertension: a systematic review and meta-analysis of epidemiological studies. Neuropsychiatr Dis Treat 2015;11:1121-30.

47 Liu M-Y, Li N, Li WA, et al. Association between psychosocial stress and hypertension: a systematic review and meta-analysis. Neurol Res 2017;39:573-80.

48 Weine S, Ukshini S, Griffith J, et al. A family approach to severe mental illness in post-war Kosovo. Psychiatry 2005;68:17-27.

49 Bogner HR, de Vries HF. Integration of depression and hypertension treatment: a pilot, randomized controlled trial. Ann Fam Med 2008:6:295-301.

50 Hankinson JL, Odencrantz JR, Fedan KB. Spirometric reference values from a sample of the general U.S. population. Am J Respir Crit Care Med 1999:159:179-87.

51 Thorat YT, Salvi SS, Kodgule RR. Peak flow meter with a questionnaire and mini-spirometer to help detect asthma and COPD in real-life clinical practice: a cross-sectional study. NPJ Prim Care Respir Med 2017;27:32.

52 Organisation mondiale de la santé. Global recommendations on physical activity for health. Genève: WHO, 2010.

53 Joint WHO-FAO Expert Consultation on Diet, Nutrition, and the Prevention of Chronic Diseases, 2002, Geneva, Switzerland. Expert consultation on diet, nutrition, and the prevention of chronic diseases, Weltgesundheitsorganisation, Fao, editors. diet, nutrition, and the prevention of chronic diseases: report of a WHO-FAO expert consultation. Geneva: World Health Organization, 2003.

54 World Health Organization, Management of Substance Abuse Team. Global status report on alcohol and health 2018, 2018. Available: http://www.who.int/substance abuse/publications/global_alcohol report/en/ [Accessed 30 Jul 2020].

55 Moser A, Stuck AE, Silliman RA, et al. The eight-item modified medical outcomes study social support survey: psychometric evaluation showed excellent performance. $J$ Clin Epidemiol 2012;65:1107-16.

56 Chaudhry S, Jin L, Meltzer D. Use of a self-report-generated Charlson comorbidity index for predicting mortality. Med Care 2005;43:607-15.

57 Rose G, McCartney P, Reid DD. Self-Administration of a questionnaire on chest pain and intermittent claudication. Br J Prev Soc Med 1977; 31:42-8.

58 European Community Respiratory Health Survey II Steering Committee. The European community respiratory health survey II. Eur Respir J 2002;20:1071-9. 
59 Bestall JC, Paul EA, Garrod R, et al. Usefulness of the medical Research Council (MRC) dyspnoea scale as a measure of disability in patients with chronic obstructive pulmonary disease. Thorax 1999;54:581-6.

60 Kroenke K, Spitzer RL, Williams JBW. The PHQ-15: validity of a new measure for evaluating the severity of somatic symptoms. Psychosom Med 2002;64:258-66.

61 Wensing M, Mainz J, Grol R. A standardised instrument for patient evaluations of general practice care in Europe. Eur J Gen Pract 2000;6:82-7.

62 Lacey SJ, Street TD. Measuring healthy behaviours using the stages of change model: an investigation into the physical activity and nutrition behaviours of Australian miners. Biopsychosoc Med 2017;11:30.

63 Spek V, Lemmens F, Chatrou M, et al. Development of a smoking abstinence self-efficacy questionnaire. Int J Behav Med 2013;20:444-9.
64 Schwarzer R, Renner B. Health-Specific self-efficacy scales. 21, 2005.

65 Hays RD, Prince-Embury S, Chen H. Rand-36 health status inventory. San Antonio, TX: The Psychological Corporation, 1998.

66 Lovibond SH, Lovibond PF. Manual for the depression anxiety stress scales. Sydney, NSW: Psychology Foundation of Australia, 1995. https://trove.nla.gov.au/work/30421447

67 Prins A, Bovin MJ, Smolenski DJ, et al. The primary care PTSD screen for DSM-5 (PC-PTSD-5): development and evaluation within a veteran primary care sample. J Gen Intern Med 2016;31:1206-11.

68 Wagnild GM, Young HM. Development and psychometric evaluation of the resilience scale. J Nurs Meas 1993;1:165-78.

69 Robins RW, Hendin HM, Trzesniewski KH. Measuring global self-esteem: construct validation of a single-item measure and the Rosenberg self-esteem scale, measuring global self-esteem: construct validation of a single-item measure and the Rosenberg self-esteem scale. Pers Soc Psychol Bull 2001;27:151-61. 
Correction: Study protocol: a prospective cohort on noncommunicable diseases among primary healthcare users living in Kosovo (KOSCO)

Obas KA, Gerold J, Bytyçi-Katanolli A, et al. Study protocol: a prospective cohort on noncommunicable diseases among primary healthcare users living in Kosovo (KOSCO). BMJ Open 2020;10:e038889. doi: 10.1136/bmjopen-2020-038889.

This article was previously published with errors. The authors noticed below errors:

On page 9, table 2 under Questionnaire column, 4th row "Short Form Health Survey version 1 (SF12v1)" has been corrected to "RAND-12 Health Status Inventory (RAND-12 HSI)".

On page 9, table 2 under Description column, fourth row "The SF12v1 is a 12-item questionnaire which measures health-related quality of life.65 Item 1 has a 5-point Likert scale from 1 (excellent) to 5 (poor); items 2 and 3 have a 3-point Likert scale from 1 (Yes, limited a lot) to 3 (No, not limited at all); Items four through seven have response choices of yes (1) and no (2). Item 8 has a 5-point Likert scale from 1 (not at all) to 5 (extremely). Items 9-12 has a 6-point Likert scale from 1 (all of the time) to 6 (none of the time). Items are divided to make physical (items $1-5,8$ ) and mental health (items 6-7, 9-12) composite scores using a norm-based method and transformed to each have a mean of 50 . The total score ranges from 0 to 100 , where a 0 score indicates the lowest level of health measured by the scales and 100 indicates the highest level of health." has been corrected to "The RAND-12 HSI is a 12-item version of the RAND-36 HSI, which measures health-related quality of life.65 The RAND-12 HSI provides estimated scores on Physical Health, Mental Health and Global Health composites of the 36-item instrument. The RAND-12 HSI uses the item response theory (IRT) and oblique (correlated) factor rotations to generate the physical and mental health summaries.65 The composite scores range from 0 to 100 , where a 0 score indicates the lowest level of health and 100 indicates the highest level of health.".

Reference 65 "Ware J, Kosinski M, Keller SD. A 12-Item short-form health survey: construction of scales and preliminary tests of eliability and validity. Med Care 1996;34:220-33." was incorrect. The correct reference is:

Hays RD, Prince-Embury S, Chen H. Rand-36 health status inventory. San Antonio, TX: The Psychological Corporation; 1998.

Open access This is an open access article distributed in accordance with the Creative Commons Attribution Non Commercial (CC BY-NC 4.0) license, which permits others to distribute, remix, adapt, build upon this work non-commercially, and license their derivative works on different terms, provided the original work is properly cited, appropriate credit is given, any changes made indicated, and the use is non-commercial. See: http://creativecommons.org/licenses/by-nc/4.0/.

C Author(s) (or their employer(s)) 2021. Re-use permitted under CC BY-NC. No commercial re-use. See rights and permissions. Published by BMJ.

BMJ Open 2021;11:e038889corr1. doi:10.1136/bmjopen-2020-038889corr1

Check for updates 